\title{
STANDING KINK MODES IN THREE-DIMENSIONAL CORONAL LOOPS
}

\author{
D. J. Pascoe and I. De Moortel \\ School of Mathematics and Statistics, University of St. Andrews, St. Andrews KY16 9SS, UK; dpascoe@mcs.st-andrews.ac.uk \\ Received 2013 March 29; accepted 2014 January 27; published 2014 March 11
}

\begin{abstract}
So far, the straight flux tube model proposed by Edwin \& Roberts is the most commonly used tool in practical coronal seismology, in particular, to infer values of the (coronal) magnetic field from observed, standing kink mode oscillations. In this paper, we compare the period predicted by this basic model with three-dimensional (3D) numerical simulations of standing kink mode oscillations, as the period is a crucial parameter in the seismological inversion to determine the magnetic field. We perform numerical simulations of standing kink modes in both straight and curved 3D coronal loops and consider excitation by internal and external drivers. The period of oscillation for the displacement of dense coronal loops is determined by the loop length and the kink speed, in agreement with the estimate based on analytical theory for straight flux tubes. For curved coronal loops embedded in a magnetic arcade and excited by an external driver, a secondary mode with a period determined by the loop length and external Alfvén speed is also present. When a low number of oscillations is considered, these two periods can result in a single, non-resolved (broad) peak in the power spectrum, particularly for low values of the density contrast for which the two periods will be relatively similar. In that case (and for this particular geometry), the presence of this additional mode would lead to ambiguous seismological estimates of the magnetic field strength.
\end{abstract}

Key words: magnetohydrodynamics (MHD) - Sun: atmosphere - Sun: corona - Sun: magnetic fields - Sun: oscillations - waves

\section{INTRODUCTION}

In the last 15 years or so, coronal seismology (Uchida 1970; Roberts et al. 1984) has developed extremely rapidly, taking full advantage of modern-day high-resolution observations. In particular, coronal seismology using standing modes of coronal loops has been used to produce estimates of the Alfvén speed or the magnetic field strength (e.g., Nakariakov et al. 1999; Nakariakov \& Ofman 2001; and, more recently, Wang et al. 2012; White \& Verwichte 2012; Nisticò et al. 2013). Basic theoretical models for slab and cylindrical geometries were produced by Edwin \& Roberts $(1982,1983)$, and further studies have considered additional effects such as curvature (Gruszecki et al. 2007; Ruderman 2009; Van Doorsselaere et al. 2009), longitudinal density variations (Erdélyi \& Verth 2007; Verth et al. 2007; Pascoe et al. 2009b), and transverse structuring (Arregui et al. 2007; Ballai 2007; Pascoe et al. 2007).

For seismological purposes, simple models are preferable because the use of more complex models makes the seismological inversions non-trivial. Hence, coronal loop oscillations are often interpreted using the basic, straight cylinder/slab models developed by Edwin \& Roberts (1982, 1983), despite the observed complex and dynamical nature of coronal loops. The applicability of this model is usually justified by assuming that, as far as practical coronal seismology is concerned, additional effects such as curvature or stratification are negligible (e.g., compared to observational uncertainties). However, for the seismologically derived parameters to be reliable, it is important to demonstrate the robustness of this simple but commonly used theoretical model or, in other words, to demonstrate that additional effects such as curvature are indeed very small. So far, however, three-dimensional (3D) numerical simulations of transverse loop oscillations excited by an external pulse have failed to support this approach. For example, Pascoe et al. (2009a) performed numerical simulations of impulsively excited MHD waves in a 3D loop model to investigate the importance of the attack angle in determining the efficiency of such excitations. In a follow-up paper, De Moortel \& Pascoe (2009) considered the same model and demonstrated that the error in the seismologically inferred magnetic field strength could be approximately $50 \%$.

The crucial parameter in these models is the period of the ("observed") oscillations; therefore, this paper is concerned with the period of oscillation of numerically modeled standing kink modes in curved 3D coronal loops, which has not yet been found to be consistent with the (theoretical) estimate for standing kink mode oscillations in straight tubes. For example, Miyagoshi et al. (2004) performed simulations for a 3D potential field and described the period of oscillation in terms of the Alfvén speed at the loop top rather than the kink speed. The interaction of fast MHD waves with 3D active regions was also modeled numerically by Ofman \& Thompson (2002) for potential and force-free fields. This work was extended to use a magnetic field from potential extrapolations by Terradas \& Ofman (2004) and Ofman $(2005,2007)$. Simulations, including an individual loop density structure, were performed by McLaughlin \& Ofman (2008). In this paper, the authors performed numerical simulations of kink modes in a straight cylinder, for which they obtained the expected period dependence (i.e., consistent with the model of Edwin \& Roberts 1983). However, when they considered a curved loop embedded in a dipole magnetic field, the period of oscillation demonstrated a significant departure from the straight tube estimate.

The goal of this paper is to use 3D numerical simulations to investigate the applicability and robustness of the commonly used, basic straight flux tube model as the simplest, most useful model desirable for practical seismological studies. Hence, analytical models incorporating more complex effects, such as curvature, are deliberately ignored because they are likely to have a much smaller impact on the seismologically inferred values of the magnetic field than the 50\% deviation found by, e.g., De Moortel \& Pascoe (2009). The paper is organized as follows: in Section 2, a straight coronal loop with internally and externally driven oscillations is considered; in Section 3, a 


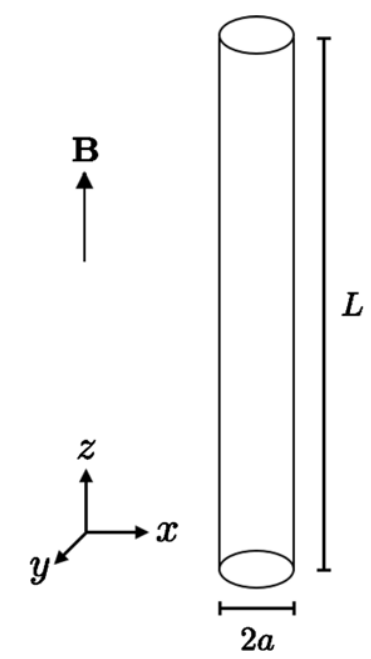

Figure 1. Model setup for the straight loop simulations. The magnetic field and loop axis are aligned with the $z$-axis.

curved 3D loop in a magnetic arcade is explored; in Section 4, a discussion of the results is provided; and in Section 5, conclusions are given.

\section{STRAIGHT FLUX TUBE}

Before we consider the oscillations of a curved coronal loop, we first obtain agreement between numerical and analytical results in a straight flux tube. The modes of oscillation for this geometry are described in detail by Edwin \& Roberts (1983).

\subsection{Model Setup}

For the simulations of straight loops, the magnetic field is uniform and aligned with the axis of the loop which is taken to be the $z$-axis (see Figure 1). The loop structure is defined as a density enhancement based on the general symmetric Epstein profile (e.g., Nakariakov \& Roberts 1995):

$$
\rho(x, y)=\left(\rho_{0}-\rho_{e}\right) \operatorname{sech}^{2}(r / a)^{p}+\rho_{e},
$$

where $r=\sqrt{x^{2}+y^{2}}, \rho_{0}$ is the internal density, $\rho_{e}$ is the external density, and $p$ determines the steepness of the profile. We choose $p=10$ in order to approximate the step-function profile used in Edwin \& Roberts (1983) while still having a finite transition from inside to outside the loop, which can be resolved numerically. Note that although the resolution (typically $\sim 400^{3}$ ) is sufficient to resolve the transition layer, it is not sufficient to resolve the resonant absorption that arises as a result of this finite boundary layer (e.g., Goossens et al. 2011). However, this paper focuses on the period of the oscillations and the damping rate is not considered in this study. Convergence tests carried out by doubling the resolution changed the measured oscillations by less than $1 \%$ and had no effect on the period of oscillation. Therefore, we can be confident that any discrepancy we might find between the period of our (numerical) loop oscillations and the theoretical model is not due to the resolution of the numerical simulations.

All simulations are performed using LARE3D (Arber et al. 2001) to solve the (nonlinear) 3D MHD equations. The boundary conditions are periodic in the $x$ - and $y$-directions, with damping layers near the edge of the numerical domain to reduce the amplitude of perturbations propagating across the boundary. The upper and lower $z$-boundaries are line-tied to simulate reflective loop footpoints.

The radius of the loop is chosen to be $a=0.5 \mathrm{Mm}$. The numerical domain has a size of $10 \times 10 \times 50 \mathrm{Mm}$, so the length of the loop is $L=50 \mathrm{Mm}$. This corresponds to the long wavelength limit with an aspect ratio of $a / L=0.01$, or normalized wavenumber $k a=0.01 \pi$ for a global mode with the wavelength $\lambda=2 L$. In this limit, the kink mode phase speed tends to the kink speed $C_{k}$; therefore, the period of oscillation for the global kink mode is $P_{k}=2 L / C_{k}$ with

$$
C_{k}=\sqrt{\frac{\rho_{0} C_{A 0}^{2}+\rho_{e} C_{A e}^{2}}{\rho_{0}+\rho_{e}}},
$$

where $C_{A 0}$ is the internal Alfvén speed and $C_{A e}$ is the external Alfvén speed. The equilibrium for the loop is defined by satisfying the condition of total pressure balance across the loop. We consider the case of thermal pressure being much lower than magnetic pressure, i.e., plasma $\beta=0.001$.

Kink oscillations are readily excited by any perturbation to the loop axis. For the case of an internal perturbation, the global kink eigenmode, or a close approximation to it, can be applied. The approximation used here is based upon, in the transverse direction, the kink eigenfunction for the Epstein profile (e.g., Cooper et al. 2003), which is symmetric at about $r=0$ and so displaces the loop axis. In the longitudinal direction, the fundamental or global loop harmonic is selected by setting

$$
v_{y}(x, y, z)=A_{0} \operatorname{sech}^{v}(r / a) \cos k z,
$$

where $A_{0}=0.001 \mathrm{Mm} \mathrm{s}^{-1}$ is the amplitude and the wavenumber is $k=\pi / L$ for the global mode. For the Epstein profile with $p=1$, the analytical solution gives $v=\left(|k| a / C_{A e}\right)$ $\sqrt{C_{A e}^{2}-(\omega / k)^{2}}$. Here, we choose $v=1.5$ as an approximation for the eigenfunction for our geometry and density profile $(p=10)$ since this gives a perturbation with suitable spatial extent, i.e., comparable to the radius of the loop.

Our choice of a low-amplitude perturbation allows us to avoid nonlinear effects, such as changes to the density profile along the loop driven by the ponderomotive force (e.g., Terradas \& Ofman 2004).

We also consider excitation by an external perturbation based on the model of a fast magnetoacoustic wave excited by some external energy release, such as a flare propagating through the corona and hitting the coronal loop from the side (e.g., Nakariakov \& Verwichte 2004). We approximate this by a plane wave with a Gaussian cross-sectional profile propagating in the $y$-direction:

$$
v_{y}(y)=A_{0} \exp -\left(\frac{y-d}{\sigma}\right)^{2}
$$

where $A_{0}=0.05 \mathrm{Mm} \mathrm{s}^{-1}, d=-2 \mathrm{Mm}$ is the initial position of the fast wave relative to the loop axis, and $\sigma=0.3 \mathrm{Mm}$ determines the spatial extent of the profile.

\subsection{Results}

Figure 2 shows the transverse velocity signal measured at the middle of a loop (i.e., $r=0, z=L / 2$ ) with a density contrast of $\rho_{0} / \rho_{e}=2$. The top panel shows the transverse velocity for the case of internal excitation. This is achieved by applying the above velocity perturbation that approximates the global kink 

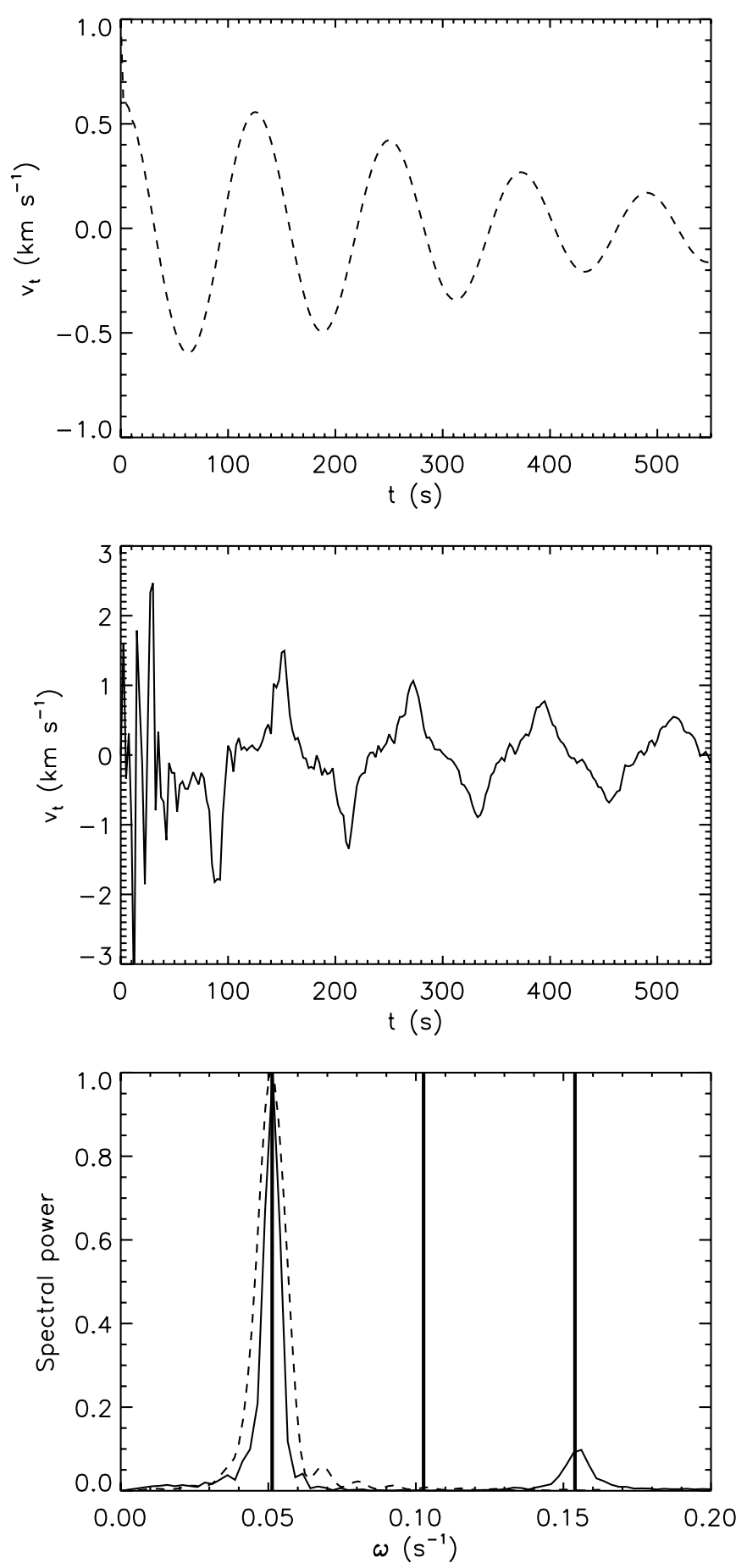

Figure 2. Transverse velocity signals $v_{t}$ for a straight tube with a density contrast of 2 excited by an internal (dashed lines) and external (solid lines) driver. The bottom panel shows the periodogram of the signals. The vertical lines denote the natural frequencies loop (longitudinal harmonics $n=1,2,3$ ).

eigenmode (Equation (3)). Although it is not an exact solution, it has the same radial and longitudinal form and is sufficiently close to the exact solution to efficiently excite a kink oscillation. The oscillation has the form of a harmonic oscillation with damping. We note again that the strong damping might be an artifact of the numerical resolution, which is not sufficient to resolve potential resonant absorption (but does not affect the period that is the main parameter we are concerned with in this study).
The middle panel of Figure 2 shows the same signal for the case of excitation by an external perturbation (Equation (4)). This has the form of a plane wave generated outside the loop that propagates toward, and through, the loop, displacing the loop axis and generating kink oscillations. Comparing the oscillation to that produced by the internal perturbation, the evident differences are that there is a much longer initial stage during which the perturbation generates the oscillation (the leaky impulsive phase during $t<50 \mathrm{~s}$ ) and the signal is no longer a single harmonic.

The bottom panel of Figure 2 shows a periodogram of the two transverse velocity signals. The vertical lines denote the natural frequencies of the loop, i.e.,

$$
\omega=k C_{k}
$$

where $k=2 \pi / \lambda$ and $\lambda=2 L / n$ for the longitudinal harmonic of order $n$. The (normalized) spectral power for both signals shows a peak corresponding to the global $(n=1)$ kink mode. For the case of internal excitation, this mode was prescribed by the applied perturbation. For the external excitation, there is also a weak oscillation generated corresponding to $n=3$. The $n=3$ harmonic is consistent with the applied perturbation and boundary conditions, i.e., having nodes at the loop footpoints prescribed by the line-tied boundaries but allowed to oscillate elsewhere. Harmonics with odd $n$ are consistent with our external perturbation, whereas those with even $n$ are unlikely due to the symmetry of the driver. (For reference, Pascoe et al. 2009a demonstrated the efficient excitation of the $n=2$ harmonic for an external driver exciting a loop with an attack angle of $\approx 45^{\circ}$.) Overall, we expect the global mode to be the most strongly excited by the plane wave due to its extended nature, as is the case in Figure 2.

In summary, for the straight model, our numerical simulations are consistent with the analytical (Edwin \& Roberts 1983) results for a straight flux tube in terms of the period of oscillation, as the error in the period is only $\approx 0.4 \%$ and, therefore, negligible for practical seismological purposes. The period is not found to depend upon the method of excitation, although the particular harmonics excited would depend upon the driver.

In reality, coronal loops are, of course, curved, but, so far, the straight flux tube model is the most commonly applied in seismology studies. Indeed, for these purposes, it makes sense to use the least complex model that can be shown to be applicable and robust to keep the seismological inversions simple. Keeping this in mind, we will now explore to what extent the estimated $P_{k}$ (from the basic, straight flux tube model of Edwin \& Roberts 1983) is consistent with the period of oscillations in curved coronal loops in our numerical simulations.

\section{CURVED CORONAL LOOPS}

We investigate a 3D loop embedded in a $2.5 \mathrm{D}$ magnetic arcade, i.e., the same model setup considered by Pascoe et al. (2009a) and De Moortel \& Pascoe (2009). In this geometry, the kink oscillation may be considered to displace the loop axis perpendicular to the plane of the loop (horizontal polarization), radially in the plane of the loop (vertical polarization), or some combination of the two.

The observational signatures of horizontal and vertical kink modes were considered by Wang et al. (2008) in their fundamental mode and second harmonic. It was found that various combinations of viewing angles and loop geometry can make it difficult to distinguish between different types of kink mode 


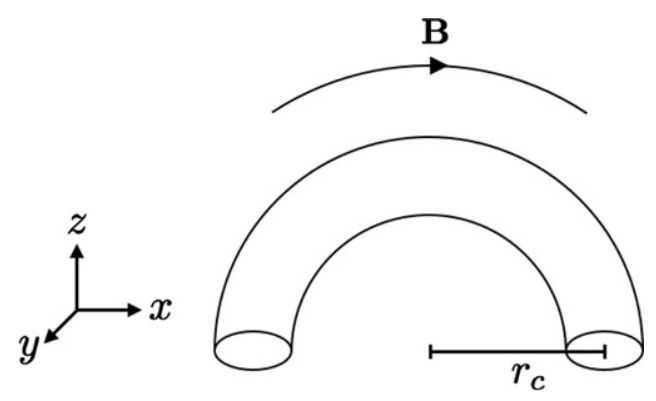

Figure 3. Model setup for the curved loop simulations. The magnetic field is a $2.5 \mathrm{D}$ arcade in the $x z$ plane.

using only a time series of images. However, this distinction is trivial for our numerical simulations, and the polarizations are expected to have almost identical periods of oscillation (Ruderman 2009; Van Doorsselaere et al. 2009).

\subsection{Model Setup}

The magnetic field is a semi-circular 2.5D arcade with $B \sim 1 / r_{p}$ (e.g., Brady et al. 2006; Pascoe et al. 2009a), where $r_{p}=\sqrt{x^{2}+z^{2}}$ is the radial distance in the plane of the arcade (see Figure 3). Within this arcade, a curved loop of (minor) radius $a$ is modeled as a density enhancement given by

$$
\rho(x, y, z)=\left(\rho_{0}-\rho_{e}\right) \operatorname{sech}^{2}(r / a)^{p}+\rho_{e},
$$

where $r=\sqrt{\left(r_{p}-r_{c}\right)^{2}+y^{2}}, r_{c}=L / \pi$ is the major loop radius. The loop length $L$, minor radius $a$, and steepness parameter $p$ are the same as for the simulations with the straight flux tube (Section 2).

The typical resolution is $1002 \times 376 \times 502$ grid points for a numerical domain of size $44 \times 7.5 \times 22 \mathrm{Mm}$. The boundary conditions are periodic in $y$ (with damping layers) and linetied for $x$ and $z$. Since the magnetic field becomes singular for $r_{p} \rightarrow 0$, we define an inner region $r_{p} \leqslant 5 \mathrm{Mm}$ for which perturbations are set to zero. This allows the magnetic field profile to stop before $r_{p}=0$ without leading to changes in the equilibrium. Since we also do not want changes in equilibrium due to curved magnetic field lines intersecting the upper $z$ - and $x$-boundaries, we similarly define the region of $r_{p}>21 \mathrm{Mm}$ where perturbations are prohibited.

\subsection{Internal and External Drivers}

We can consider the same internal and external (plane-wave) drivers as in Section 2, with the minor modification that the internal driver now considers the curvature of the loop, i.e.,

$$
v_{y}(x, y, z)=A_{0} \operatorname{sech}^{v}(r / a),
$$

where $r=\sqrt{\left(r_{p}-r_{c}\right)^{2}+y^{2}}$, as for the density profile in Equation (6).

Figure 4 shows the transverse velocity signals $v_{t}$ (top panel) at the loop apex for a curved coronal loop with a density contrast of 2. The dashed and solid lines correspond to internal and plane-wave drivers, respectively. The bottom panel shows the corresponding periodograms. As with the straight loop, for the internal driver, we obtain a peak in spectral power at the frequency corresponding to the global kink mode (dashed vertical line). However, for the external driver, we now see a different behavior. There is a peak at the global kink mode frequency $\omega_{k}=2 \pi / P_{k}$, but also a larger second peak at a
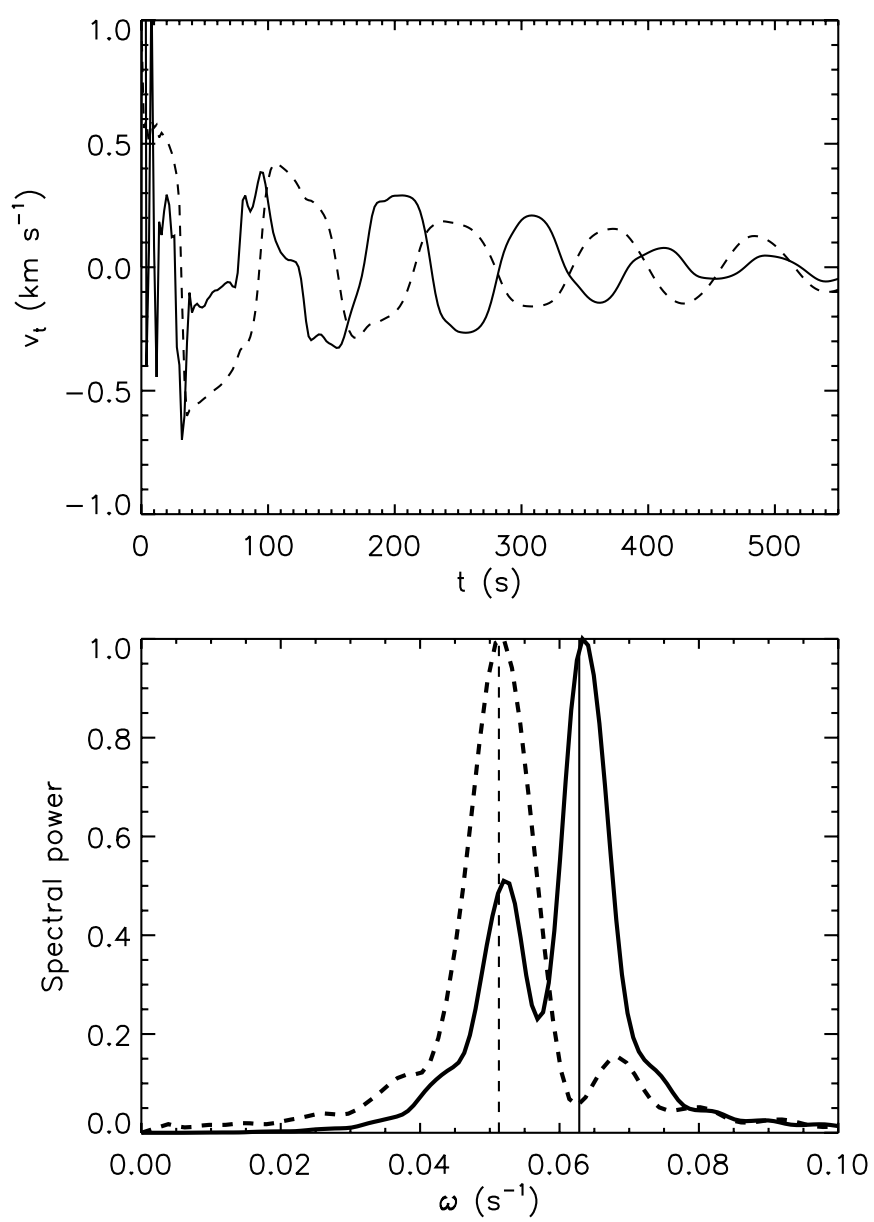

Figure 4. Transverse velocity signals $v_{t}$ at the apex of a curved loop with a density contrast of 2 excited by an internal (dashed) and external (solid) driver. The bottom panel shows the periodogram of the signals. The vertical lines represent $\omega_{k}$ (dashed) and $\omega_{A}$ (solid).

higher frequency. This peak occurs at a frequency determined by the (external) Alfvén speed rather than the kink speed, i.e., $\omega_{A}=2 \pi / P_{A}$, where $P_{A}=2 L / C_{A e}$ and $C_{A e} \propto 1 / r_{p}$ (solid vertical line). The small offset $(\approx 2 \%)$ between the locations of the peaks in the power spectrum (of the curved numerical loop oscillations) and the predicted values of $\omega_{A}$ and $\omega_{k}$ (based on the straight flux tube model) is of the order of the effect of curvature on the kink mode period predicted by Van Doorsselaere et al. (2009).

Figure 5 shows the variation of the spectral amplitude for $\omega_{A}$ with angle $\theta$ around the loop, where the loop footpoints are at $\theta=0^{\circ}$ and $180^{\circ}$ and the apex is at $\theta=90^{\circ}$. The crosses and squares correspond to the behavior for $v_{t}$ and the radial velocity signal $v_{r}$, respectively, with each normalized to the maximum spectral amplitude for $v_{t}$. Although the driver is initially a plane wave, it undergoes significant refraction due to the non-uniform magnetic field strength and Alfvén speed. Therefore, the loop has an oscillation in the radial direction, i.e., a vertically polarized global kink mode. The period of this mode is consistent with $P_{k}$ since both horizontally and vertically polarized modes have the same frequency. Both profiles show the expected global mode structure, indicated by the sinusoidal curves.

In addition to the internal and plane-wave drivers, we can also consider an external driver generated by a localized impulsive energy deposition, as in Pascoe et al. (2009a), who investigated 


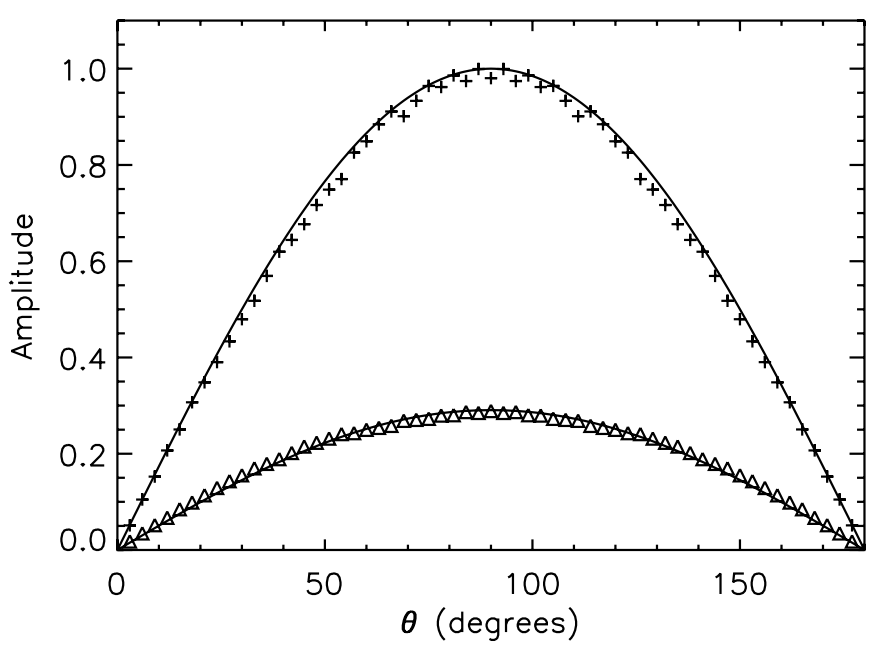

Figure 5. Variation of the spectral amplitude at $\omega=\omega_{k}$ with angle $\theta$ around the loop. The crosses and squares correspond to the $v_{t}$ and $v_{r}$ signals, respectively.

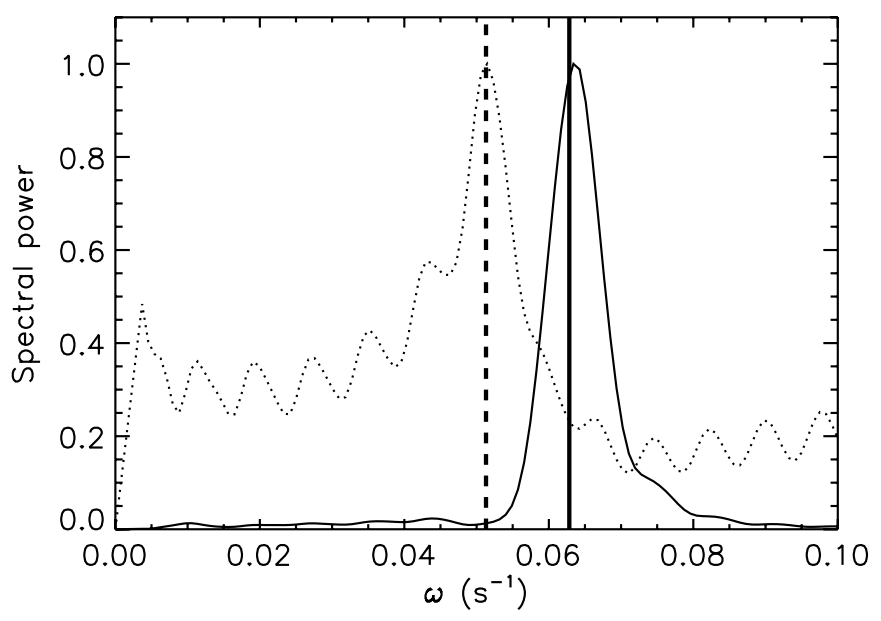

Figure 6. Simulation for excitation of loop oscillations using a pulse driver. The solid and dotted curves represent the periodograms for the signals in $v_{t}$ and $v_{r}$, respectively, at the loop apex. The vertical lines represent $\omega_{k}$ (dashed) and $\omega_{A}$ (solid).

the case of a localized driver situated at the height of the loop apex in detail. The behavior is similar to the plane wave described above, i.e., the spectra for transverse loop oscillations show two distinct peaks corresponding to $\omega_{k}$ and $\omega_{A}$. Figure 6 shows the periodogram for the transverse and radial velocity signals (solid and dotted lines, respectively) for a simulation with the pulse located at $\left(0, d, r_{c}-d\right)$. This driver appears to generate the kink oscillation only in the radial velocity perturbations because the pulse is located lower than the loop apex, making it less efficient at exciting transverse oscillations than the plane-wave driver. A peak only occurs at $\omega_{k}$ for the radial velocity oscillations.

\subsubsection{High Density Contrast Loop}

Figure 7 shows the periodograms for the transverse (solid) and radial (dotted) velocity signals at the loop apex for simulations with loop density contrasts of $\rho_{0} / \rho_{e}=2$ (top panel; see also Section 3.2) and 10 (bottom panel). The horizontally and vertically polarized modes have the same frequency; however, the radial velocity signal again does not contain the second peak in frequency corresponding to the arcade oscillation. For both the high and low density contrasts, we see that the
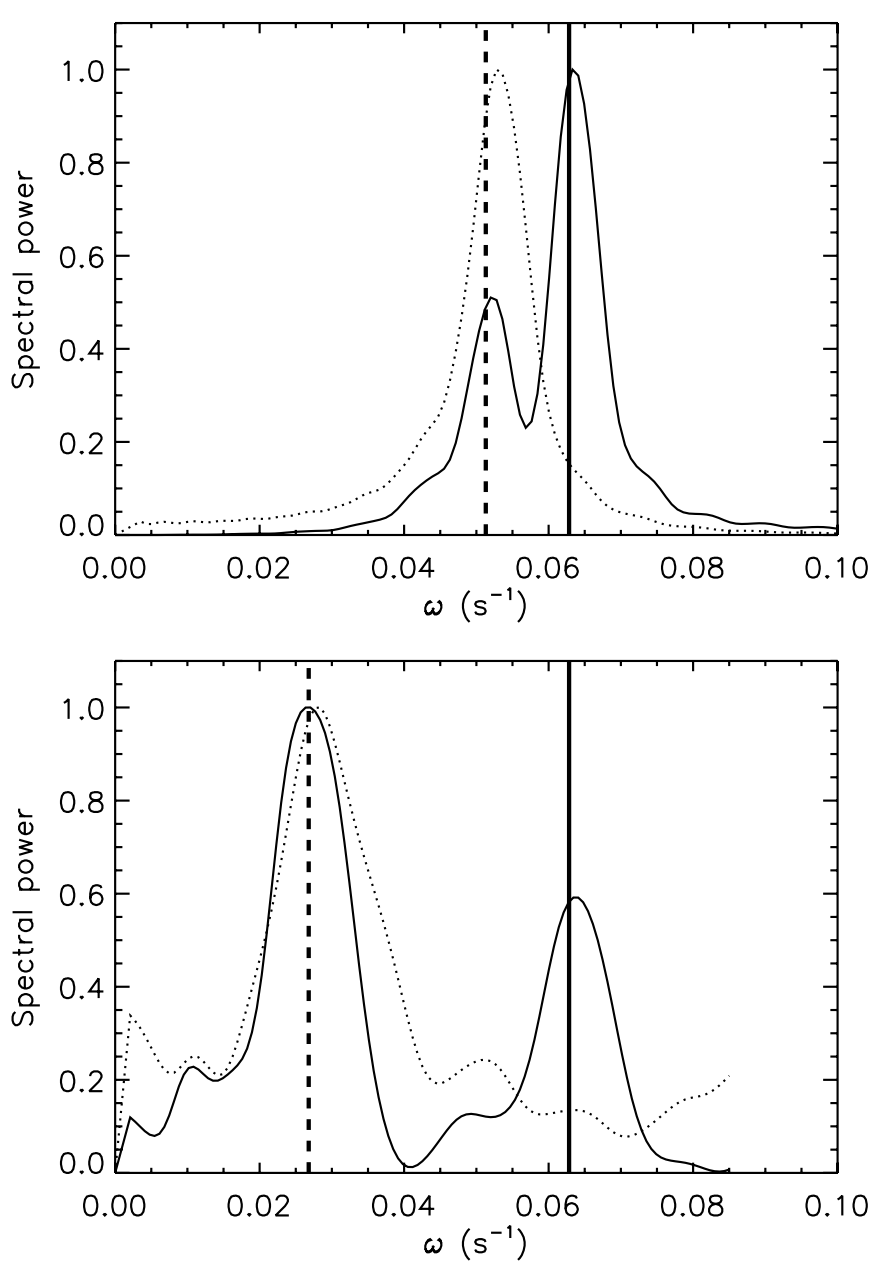

Figure 7. Simulation for a loop with density contrasts (top) $\rho_{0} / \rho_{e}=2$ and (bottom) 10 excited by a plane-wave driver. The solid and dotted curves represent the periodograms for the signals in $v_{t}$ and $v_{r}$, respectively, at the loop apex. The vertical lines represent $\omega_{k}$ (dashed) and $\omega_{A}$ (solid).

transverse velocity signal contains two components, close to the frequencies of $\omega_{k}$ (dashed vertical line) and $\omega_{A}$ (solid vertical line), whereas the radial velocity signal only appears to contain the $\omega_{k}$ component. For higher density contrasts, the kink frequency $\omega_{k}$ is smaller, while $\omega_{A}$ remains constant. Consequently, the two peaks in the spectral profile are separated for increasing density contrast.

\subsubsection{Simulation with no Loop Structure}

Here, we consider the oscillations generated by the planewave driver propagating through our two-dimensional (2D) magnetic field with no density structure, i.e., $\rho_{0} / \rho_{e}=1$. Figure 8 shows the periodogram for the transverse and radial velocity signals (solid and dotted lines, respectively) for a simulation with uniform density. The signals are taken at the same location as in previous simulations; however, no loop is currently present at that location.

Both the transverse and radial signals have a single period of oscillation corresponding to $P_{A}=2 L / C_{A}$, i.e., the period is determined by the loop length and the Alfvén speed. Note that the Alfvén speed of $C_{A}$ is equal to the external Alfvén speed of $C_{A e}$ used earlier, but is now the only characteristic speed in our model since the density is uniform and $\beta$ is small. The bottom panel of Figure 8 shows the variation of the spectral amplitude for $\omega_{A}$ with angle $\theta$ around the loop, as in Figure 5 . The global mode structure is once again evident, so we have 

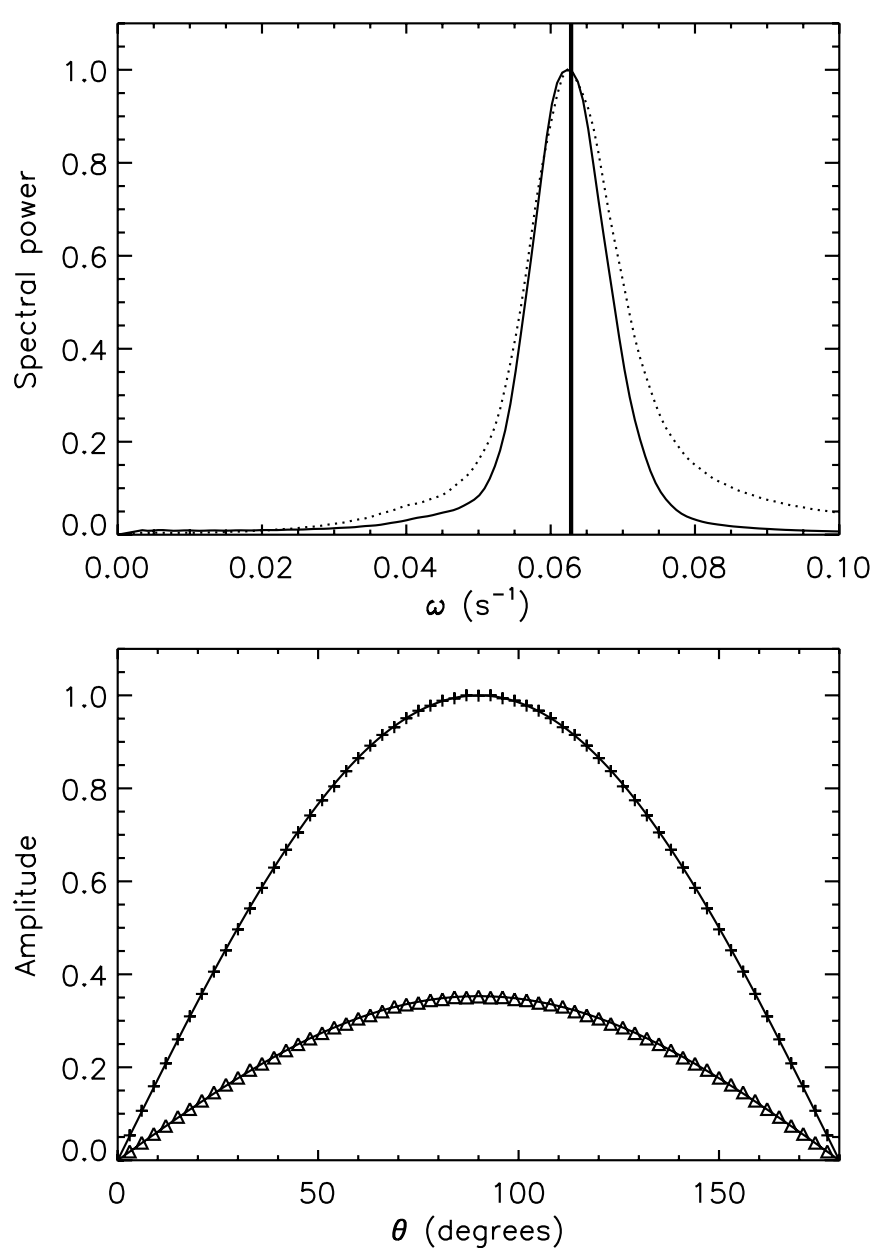

Figure 8. Simulation for the plane-wave driver in a plasma with uniform density, i.e., no loop structure. The solid and dotted curves in the top panel represent the periodograms for the signals in $v_{t}$ and $v_{r}$, respectively, taken at the same location as Figure 7. The dashed vertical line represents $\omega_{A}$. The bottom panel shows the variation of the spectral amplitude at $\omega=\omega_{A}$ with angle $\theta$ around the loop. The crosses and squares correspond to the $v_{t}$ and $v_{r}$ signals, respectively.

a global standing Alfvén mode: the oscillation has a global standing mode structure $(k=\pi / L)$ and a period of oscillation that satisfies the Alfvén wave dispersion relation $\omega=C_{A} k$. This mode is not seen in the case of a straight magnetic field without a loop structure because the uniform field does not define any flux surfaces. The curved magnetic arcade reduces the symmetry and requires a varying field strength (which varies perpendicular to the axis of the field) and allows flux surfaces to be defined.

Note that the radial oscillation at $P_{A}$ was not seen in the previous simulations. This suggests that when the loop is present, the radial oscillation tends to be dominated by the kink mode, possibly due to the extended and collective nature of the kink mode eigenfunction, i.e., the kink eigenmode extends over multiple flux surfaces and couples them together to create a coherent oscillation with a single "averaged" frequency of $\omega_{k}$. This produces a spatially (radially) robust signal, in contrast with the Alfvén mode which has a different frequency, $\omega_{A}(r)$, for each individual flux surface (and which also leads to phase mixing of Alfvén waves).

\section{DISCUSSION}

Figure 4 of De Moortel \& Pascoe (2009) shows a periodogram with a peak spectral amplitude at a period significantly less than

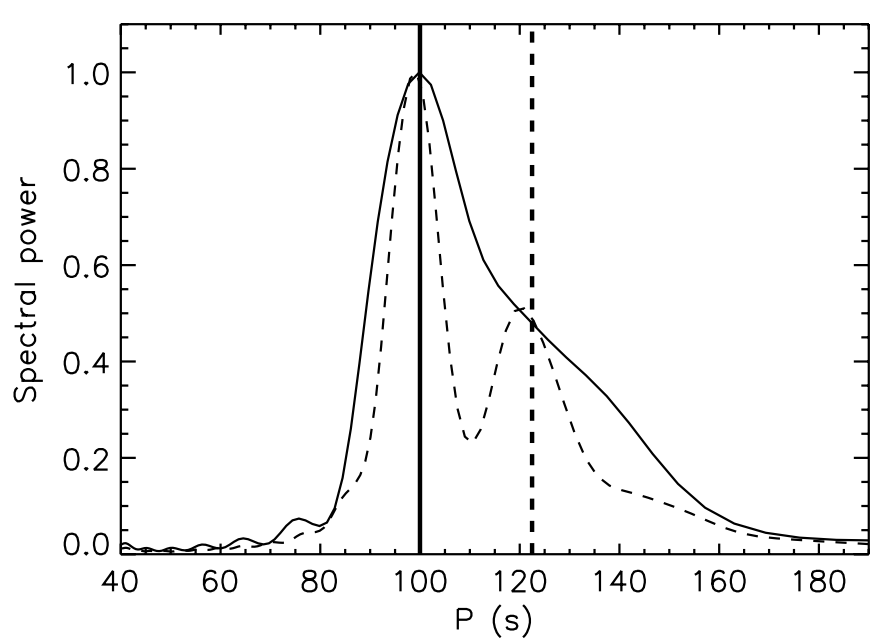

Figure 9. Reproduction of Figure 4 in De Moortel \& Pascoe (2009) (solid curve). The spectral power has a broad peak at a period of $P_{A}=2 L / C_{A e}$ (solid vertical line). A longer data series (dashed curve) resolves the peak at $P_{k}=2 L / C_{k}$ (dashed vertical line).

$P_{k}=2 L / C_{k}$. There is an asymmetric broadening to the peak on the high period side. Here, we demonstrate that this asymmetric broadening is in fact an unresolved version of the double-peak structure shown by the dashed line in Figure 4 of this paper; in Figure 9, the dashed curve is the same signal as in our Figure 4 and contains $\approx 6$ periods of oscillation. The solid curve is the periodogram of the same signal, but only considers $\approx 3$ periods of oscillation. For the shorter time series, the dominant peak at $P_{A}=2 L / C_{A e}$ (vertical dashed line) is resolved, but the peak at $P_{k}$ (vertical solid line) is unresolved. Note that even in the case of the two peaks being resolved, the damping of the oscillations leads to finite peak widths. The agreement of the peaks with the predicted frequencies of $\omega_{k}$ and $\omega_{A}$ (see also the previous figures) is $\approx 2 \%$. Our simulations therefore cannot rule out the possibility of curvature having a small effect on the period of oscillation of kink modes (e.g., Van Doorsselaere et al. 2009), but they do explain the much larger discrepancy reported in De Moortel \& Pascoe (2009).

It is possible that a similar effect could account for other 3D numerical studies of curved loop oscillations also obtaining a period of oscillation not consistent with $P_{k}$ (as determined from the straight flux tube model). For example, McLaughlin \& Ofman (2008) considered oscillations excited by a velocity pulse launched from the boundary similar to our plane-wave driver. These authors considered a dipole magnetic field rather than the arcade studied here; however, it shares the property of being a symmetric but non-uniform field, so Alfvén waves may be supported by the flux surfaces defined by the field.

Miyagoshi et al. (2004) excited oscillations by applying a velocity field to the top of their loop in a way similar to our internal driver. For our internal excitation simulations, we find the period of oscillation is consistent with $P_{k}$, whereas Miyagoshi et al. (2004) described the period of oscillation in terms of the Alfvén speed at the loop top and found that $P \propto \rho_{0}^{0.33}$. Figure 10 shows the dependence on the period of oscillation on the density contrast. The solid curve is the dependence of the global kink mode period for our simulations, i.e., $P_{k}=2 L / C_{k}$ and Equation (2) with $L=50 \mathrm{Mm}$. The symbols represent the analytical kink period $P_{k}$ for the density contrast ratios considered by Miyagoshi et al. (2004). The dashed line represents the power law $P \propto \rho_{0}^{0.33}$, which can be fitted reasonably through a limited parameter range such 


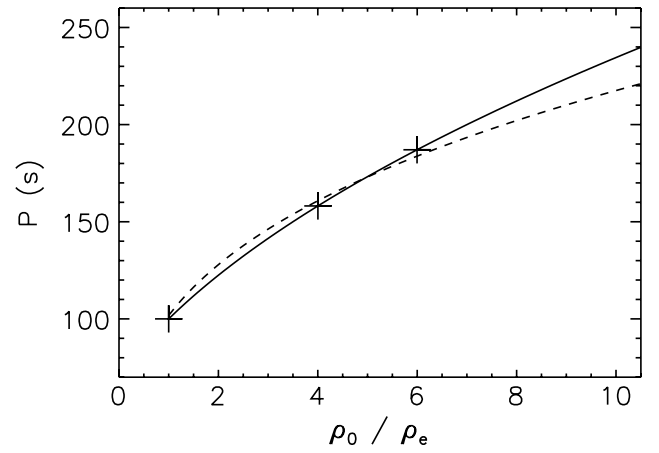

Figure 10. Dependence of period of oscillation of the global standing kink mode upon density contrast. The dashed line represents a power law of the form $P \propto \rho_{0}^{0.33}$, suggested by Miyagoshi et al. (2004), which may approximate the kink mode behavior for the narrow range of parameters considered by those authors (symbols).

as the one they considered. Therefore, it is possible that the period of oscillation found by Miyagoshi et al. (2004) is actually consistent with the estimate based on straight tube theory; however, to confirm this, a more detailed analysis of their data is required.

\section{CONCLUSIONS}

We have performed numerical simulations of curved 3D coronal loops and demonstrated that the period of oscillation for kink modes (in the long wavelength limit) is sufficiently determined by the loop length and kink speed to support the use of straight tube theory for practical coronal seismology. This applies to both horizontally and vertically polarized kink modes and does not depend upon the method of excitation. However, for a symmetrical magnetic field, such as the 2D magnetic arcade used in Section 3, it is also possible for an external driver to efficiently generate arcade oscillations for which the period is essentially determined by the loop length and the external Alfvén speed. When a low number of oscillations is considered, these two periods might not be distinctly resolved, but they result in a broad peak in the power spectrum, particularly for low values of the density contrast for which the two periods will be relatively similar. This accounts for the deviation in the period of oscillation reported by De Moortel \& Pascoe (2009) and, possibly, also the departures of the period from the straight tube estimate reported by other authors. It may be necessary for this to be taken into account in similar numerical simulations performed with a symmetrical setup. Although the effect mainly arises due to the symmetry in our simulations, it could be relevant to observations of highly symmetrical configurations, such as those present in post-flare coronal loops (e.g., Verwichte et al. 2004) and isolated dipole-like active regions, particularly because the number of observed oscillations tends to be low (e.g., Aschwanden et al. 2002). In such a case, the two periods are unlikely to be resolved and the single broad peak in the power spectrum would lead to ambiguous seismological estimates of the magnetic field strength. However, the importance of this effect for observations of more complex active regions requires further investigation.

I.D.M. acknowledges support from a Royal Society University Research Fellowship. The computational work for this paper was carried out at the joint STFC and SFC (SRIF)-funded cluster at the University of St Andrews (UK). The research leading to these results has also received funding from the European Commissions Seventh Framework Programme (FP7/2007-2013) under the grant agreement SOLSPANET (project No. 269299; www.solspanet.eu/solspanet). The authors would like to thank the referee for their helpful comments, which have improved the paper.

\section{REFERENCES}

Arber, T. D., Longbottom, A. W., Gerrard, C. L., \& Milne, A. M. 2001, JCoPh, 171,151

Arregui, I., Andries, J., Van Doorsselaere, T., Goossens, M., \& Poedts, S. 2007, A\&A, 463, 333

Aschwanden, M. J., De Pontieu, B., Schrijver, C. J., \& Title, A. 2002, SoPh, 206, 99

Ballai, I. 2007, SoPh, 246, 177

Brady, C. S., Verwichte, E., \& Arber, T. D. 2006, A\&A, 449, 389

Cooper, F. C., Nakariakov, V. M., \& Williams, D. R. 2003, A\&A, 409, 325

De Moortel, I., \& Pascoe, D. J. 2009, ApJL, 699, L72

Edwin, P. M., \& Roberts, B. 1982, SoPh, 76, 239

Edwin, P. M., \& Roberts, B. 1983, SoPh, 88, 179

Erdélyi, R., \& Verth, G. 2007, A\&A, 462, 743

Goossens, M., Erdélyi, R., \& Ruderman, M. S. 2011, SSRv, 158, 289

Gruszecki, M., Murawski, K., Solanki, S. K., \& Ofman, L. 2007, A\&A, 469, 1117

McLaughlin, J. A., \& Ofman, L. 2008, ApJ, 682, 1338

Miyagoshi, T., Yokoyama, T., \& Shimojo, M. 2004, PASJ, 56, 207

Nakariakov, N. M., Ofman, L., DeLuca, E., Roberts, B., \& Davila, J. M. 1999, Sci, 285, 862

Nakariakov, V. M., \& Ofman, L. 2001, A\&A, 372, L53

Nakariakov, V. M., \& Roberts, B. 1995, SoPh, 159, 399

Nakariakov, V. M., \& Verwichte, E. 2004, A\&G, 45, 040000

Nisticò, G., Nakariakov, V. M., \& Verwichte, E. 2013, A\&A, 552, A57

Ofman, L. 2005, AdSpR, 36, 1572

Ofman, L. 2007, ApJ, 655, 1134

Ofman, L., \& Thompson, B. J. 2002, ApJ, 574, 440

Pascoe, D. J., De Moortel, I., \& McLaughlin, J. A. 2009a, A\&A, 505, 319

Pascoe, D. J., Nakariakov, V. M., \& Arber, T. D. 2007, SoPh, 246, 165

Pascoe, D. J., Nakariakov, V. M., Arber, T. D., \& Murawski, K. 2009b, A\&A, 494, 1119

Roberts, B., Edwin, P. M., \& Benz, A. O. 1984, ApJ, 279, 857

Ruderman, M. S. 2009, A\&A, 506, 885

Terradas, J., \& Ofman, L. 2004, ApJ, 610, 523

Uchida, Y. 1970, PASJ, 22, 341

Van Doorsselaere, T., Verwichte, E., \& Terradas, J. 2009, SSRv, 149, 299

Verth, G., Van Doorsselaere, T., Erdélyi, R., \& Goossens, M. 2007, A\&A, 475,341

Verwichte, E., Nakariakov, V. M., Ofman, L., \& Deluca, E. E. 2004, SoPh, 223,77

Wang, T., Ofman, L., Davila, J. M., \& Su, Y. 2012, ApJL, 751, L27

Wang, T. J., Solanki, S. K., \& Selwa, M. 2008, A\&A, 489, 1307

White, R. S., \& Verwichte, E. 2012, Aap, 537, A49 